\title{
HIERARCHICAL MULTIPLE MODELS ADAPTIVE DECOUPLING CONTROLLER APPLIED TO THE WIND TUNNEL SYSTEM
}

\author{
Xin Wang ${ }^{1} \dagger$, Shao-Yuan $\mathrm{Li}^{2}$ and Zhong-Jie Wang ${ }^{3}$ \\ 1. Institute of Information \& Control Technology, Center of Electrical \& Electronic \\ Technology, Shanghai Jiao Tong University, Shanghai, P.R.China, 200030 \\ †Corresponding Author Email: wangxin26@sjtu.edu.cn \\ 2. Institute of Automation, Shanghai Jiao Tong University, Shanghai, 200030 \\ 3. Department of Control Science \& Engineering, Tongji University, 200092
}

\begin{abstract}
For the biggest wind tunnel in Asia, during the aerodynamic research on the scale models, it is difficult to keep the Mach number in the test section and the stagnation pressure constant strictly because the interaction is strong, the operation conditions change abruptly and the transient response's requirements are high. To cope with these problems, a Hierarchical Multiple Models Adaptive Decoupling Controller (HMMADC) is presented in this paper. The controller is composed of multiple fixed controller models and two adaptive controller models. Multiple models are used to improve the transient response of the wind tunnel. Hierarchical structure is presented to reduce the number of the fixed models greatly. Adaptive Decoupling Controller not only decouples the system dynamically but also places the poles of the closed loop system arbitrarily. The significance of the proposed method is that it is applicable to a MIMO system with a much small number of models. The global convergence is obtained. Finally, several simulation examples in a wind tunnel experiment are given to show both effectiveness and practicality of the proposed method. Copyright (C) 2005 IFAC
\end{abstract}

Keywords: hierarchical, multiple models, adaptive, wind tunnel

\section{INTRODUCTION}

A $2.4 \mathrm{~m} \times 2.4 \mathrm{~m}$ injector driven transonic wind tunnel in China Aerodynamics Research and Development Center (CARDC) is the biggest wind tunnel in Asia (Zhang et al., 1997). It is used for aerodynamic research on scale models, which is very important for national defense and civil aviation. Aerodynamic research data of scale models are measured at a given Mach number with a constant stagnation pressure. It is required that in the initial stage, the response time should be no longer than 7.0 seconds; in the experiment stage, the steady state tracking errors are within $0.2 \%$ in 0.8 second and the overshoot should be avoided (Yu and Zhang, 1997). Recently several controllers are designed to satisfy the transient response's requirement above. According to a $1.5 \mathrm{~m}$ wind tunnel (FFA- T1500) in Sweden, several separate SISO models are used to control it (Nelson, 1989). For a $1.6 \mathrm{~m}$ x $2 \mathrm{~m}$ wind tunnel in Netherlands, it is regarded as a second-order system and a PID controller is given (Pels, 1989). Later a predictive controller is designed to control the Mach number in this wind tunnel with the angle of attack changing (Soeterboek et al., 1991). In USA, a system of self-organization neural networks are developed and tested to cluster, predict and control the Mach number of a 16-foot wind tunnel in NASA (Motter and Principe, 1997). However, if the descriptions for the aerodynamics of a wind tunnel are different with the size of a wind tunnel, the controller should be also different. For the $2.4 \mathrm{~m} \times 2.4 \mathrm{~m}$ transonic wind tunnel in CARDC, two SISO stable linear reduced order models are established and two PID controllers are designed to control the Mach number and the stagnation total pressure respectively (Yu and Zhang, 1997). But when the Mach number in the test section varies from 0.3 to 1.2 , the interaction becomes stronger and a multivariable decoupling controller is needed (CARDC, 2002). In (Zhang et al., 1997), two feedforward static decouplers with four fixed PI controllers are designed to solve this problem. But when the Mach number steps from 0.3 to 0.4 , $0.5, \ldots, 1.2$, the parameters of the wind tunnel will jump accordingly. The poor transient response 
cannot satisfy the high requirements of the wind tunnel above. So some special controller structure and control algorithms are needed.

To solve this problem, some multiple models adaptive controllers (MMAC) are designed to improve the transient response (Narendra and Xiang, 2000; Wang et al., 2002). One adaptive model, one reinitialized adaptive model and lots of fixed models are used to cover the region where the parameters change. For example, about 300 models are needed to cover the region where only one parameter changes (Narendra et al., 1995). The number of the models is so large that it increases the calculation time, which affects the selection of the sampling period. To reduce the huge number of models needed in MMAC, Localization, Moving Bank and other methods are presented (Zhivoglyadov et al., 2000; Maybeck et al., 1987). However, these methods can only reduce a small number of the models, which can't solve this problem essentially.

In this paper, a Hierarchical Multiple Models Adaptive Decoupling Controller (HMMADC) is presented to improve the wind tunnel's transient response. Multiple models are used to improve the transient response of the wind tunnel. Hierarchical structure is presented to reduce the number of the fixed models greatly. Adaptive Decoupling Controller not only decouples the system dynamically but also places the poles of the closed loop system arbitrarily. The proof of the global convergence is obtained. Several simulation examples in the wind tunnel experiment illustrate that the HMMADC can improve the transient response of the wind tunnel, which satisfies the requirements.

\section{DESCRIPTION OF THE SYSTEM}

\subsection{Description of the wind tunnel}

The $2.4 \mathrm{~m} \times 2.4 \mathrm{~m}$ wind tunnel is an intermittent wind tunnel constructed for the aerodynamic research aim by CARDC. It is a closed-circuit interjector driven transonic tunnel and used for testing scale models, mostly of airplanes, in the speed region of 0.3 to 1.2 (see fig.1). The interjector is used to realize high Mach numbers with the limited amount of air storage while the Reynolds number can be increased in order to decrease the influence of model factors on the measurements. At the initial stage of the aerodynamic experiment, the main control hydraulic servo valve is opened and air is allowed to flow from storage bottle into the tunnel. Part of the air is let out through the main exhaust hydraulic servo valve; the other is injected into the tunnel by the injector. After the stable flowing field is established, the experiment proceeds. It has more than 40 operation cases. One of these cases is as follows (Zhang et al., 1997). At the initial stage of the experiment, the main control hydraulic servo valve is tuned to give the initial value of the Mach number in the test section with the main exhaust hydraulic servo valve and the choke finger at the preset position. After the stable flowing field is established, the exhaust hydraulic servo valve is tuned to keep the stagnation total pressure to be 1.5 , and the choke finger makes the Mach number in the test section vary with $\Delta M=0.1$ from 0.3 to 1.2 , while the main control hydraulic servo valve is controlled to ensure the injector total pressure constant and compensates for the loss of the air storage pressure. When the Mach number in the test section is larger than 0.8 , the choke finger is opened at its maximal position and the plenum exhaust valve is used to tune the Mach number in the test section correspondingly.

Because the experiment time of the wind tunnel is limited, the control objective is to ensure that (Zhang et al., 1997).

(1) In the initial stage, the time to establish the stable flowing field at the beginning of the initial Mach number in the test section is not larger than 7.0 seconds, the Mach number tracking error is within $0.2 \%$.

(2) In the experiment stage, when the Mach number in the test section is varying with $\Delta M=0.1$, the steady state tracking errors are within $0.2 \%$ in 0.8 second.

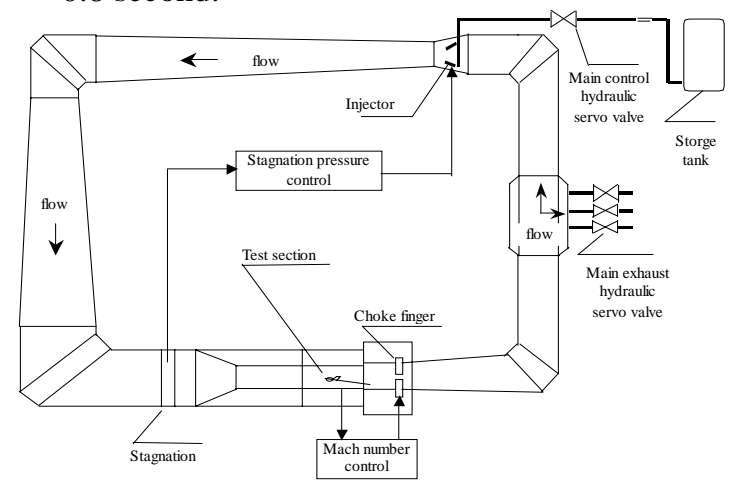

Fig. 1 The structure of the transonic wind tunnel

\subsection{The plant model}

On account of the safety of the wind tunnel system, only step signal is permitted to be inputted to identify the system. Because the Mach number in the test section varies 10 times and in each case two experiments are needed, there should be 20 experiments needed to be done theoretically. But in practice, only four experiments are permitted. So two models are obtained when the Mach number in the test section equals to 0.3 and 1.2 (CARDC, 2002). From these two particular models, the linear reduced-order model of the wind tunnel can be established according to each Mach number as follows

$\left[\begin{array}{l}y_{1}(s) \\ y_{2}(s)\end{array}\right]=\left[\begin{array}{cc}-\frac{\beta_{1}}{\alpha_{1} s+1} e^{-0.4 s} & -\frac{\beta_{2}}{\left(\alpha_{2} s+1\right)^{2}} e^{-0.4 s} \\ -\frac{\beta_{3} s+1}{\left(\alpha_{3} s+1\right)^{2}} e^{-0.4 s} & \frac{\beta_{4}}{\alpha_{4} s+1} e^{-0.4 s}\end{array}\right] \cdot\left[\begin{array}{l}u_{1}(s) \\ u_{2}(s)\end{array}\right]$,

where $y_{1}(s), y_{2}(s), u_{1}(s), u_{2}(s)$ are the Mach number in the test section, the stagnation total pressure, the choke finger opening and the main exhaust hydraulic servo valve respectively. $\alpha_{i}, \beta_{i}$ are parameters. and satisfy $\alpha_{i} \in\left[\alpha_{i \min }, \alpha_{i \max }\right]$, 
$\beta_{i} \in\left[\beta_{i \min }, \beta_{i \max }\right]$. Select the sampling period as 0.1 second. Then the linear discrete time multivariable minimum phase system is described as

$\left(\boldsymbol{I}+\boldsymbol{A}_{1} z^{-1}+\boldsymbol{A}_{2} z^{-2}\right) \boldsymbol{y}(t)=\left(\boldsymbol{B}_{0}+\boldsymbol{B}_{1} z^{-1}\right) \boldsymbol{u}(t-4)+\boldsymbol{d}$

The system is of second order and the time delay equals to 4 .

When the Mach number varies, the parameters of the system change accordingly. So the system can be viewed as a linear MIMO discrete-time system, which admits DARMA representation of the form

$$
\boldsymbol{A}\left(t, z^{-1}\right) \boldsymbol{y}(t)=\boldsymbol{B}\left(t, z^{-1}\right) \boldsymbol{u}(t-k)+\boldsymbol{d}(t),
$$

where $\boldsymbol{u}(t), \boldsymbol{y}(t)$ are the $n \times 1$ input, output vectors respectively and $\boldsymbol{d}(t)$ is a $n \times 1$ vector denoting the steady state disturbance. $\boldsymbol{A}\left(t, z^{-1}\right), \boldsymbol{B}\left(t, z^{-1}\right)$ are polynomial matrixes in the unit delay operator $z^{-1}$ and $\boldsymbol{B}_{0}(t)$ is nonsingular, for any $t$.

The system satisfies the assumptions as follows:

(1) The system parameters are time variant with infrequent large jumps. The period between two adjacent jumps is large enough to keep the jumping parameters constant.

(2) $\boldsymbol{\Phi}(t)=\left[-\boldsymbol{A}_{1}(t), \cdots ; \boldsymbol{B}_{0}(t), \cdots ; \boldsymbol{d}(t)\right]$ is the system model, which changes, in a compact set $\boldsymbol{\Sigma}$. (3) The upper bounds of the orders of $\boldsymbol{A}\left(t, z^{-1}\right), \boldsymbol{B}\left(t, z^{-1}\right)$ and the time delay $k$ are known a prior;

(4) The system is minimum-phase.

From assumption 1), $\boldsymbol{A}_{i}(t), \boldsymbol{B}_{j}(t), \boldsymbol{d}(t)$ are piecewise constant (time variant system with infrequent large jumping parameters). During the period when no jumps happen, (1) can be rewritten as

$$
\boldsymbol{A}\left(z^{-1}\right) \boldsymbol{y}(t+k)=\boldsymbol{B}\left(z^{-1}\right) \boldsymbol{u}(t)+\boldsymbol{d}
$$

without loss of the generality.

\section{HIERARCHICAL MULTIPLE MODELS ADAPTIVE DECOUPLING CONTROLLER}

A HMMADC is extended from the conventional MMADC directly (Wang et al., 2004). It is composed of fixed controller models, one free-running adaptive controller model and one reinitialized adaptive controller model. The fixed controller models adopt the hierarchical structure to reduce the number of the fixed models.

\subsection{Hierarchical Principle of HMMADC}

To reduce the number of the fixed models, a hierarchical structure with $l$ levels is adopted (see fig.2).

(1) Utilizing the prior information, the set $\Sigma$, where the parameters of the system vary, is partitioned into $m_{1}$ subsets $\sum_{1, s},\left(s=1, \cdots, m_{1}\right)$. In each subset, the center $\underset{1, s}{\boldsymbol{\Phi}}$ and its radius $\underset{1, s}{r}$ are designed to satisfy that For any $\boldsymbol{\Phi} \in \boldsymbol{\Sigma}_{1, s}$, $\|\boldsymbol{\Phi}-\underset{1, s}{\boldsymbol{\Phi}}\| \leq \underset{1, s}{r}$. So the centers $\underset{1, s}{\boldsymbol{\Phi}}, \quad s=1, \cdots, m_{1}$ compose the level 1 fixed model set which covers the system parameter set with their neighbors entirely.

(2) According to the switching index, the best model in level 1 is selected as $j_{1}$.

(3) Based on the best model $j_{1}$ in level 1 and use the partition method presented above similarly, $m_{2}$ centers are set up to compose the level 2 fixed model set on line dynamically, which covers the model $j_{1}$ with their neighbors entirely.

(4) According to the switching index, the best model in level 2 is selected as $j_{2}$.

(5) Similarly, the best model in the last level i.e. level $l$ is selected as $j_{l}$, which is also the best model among all the fixed models.

(6) At last, in level $l+1$, a free running adaptive model and a reinitialized adaptive model are added in. According to the switching index, the best model is selected among these three models above. The free running adaptive model is used to guarantee the stability of the wind tunnel while the reinitialized adaptive model's initial value can be set to be that of the best model selected to improve the transient response of the wind tunnel.

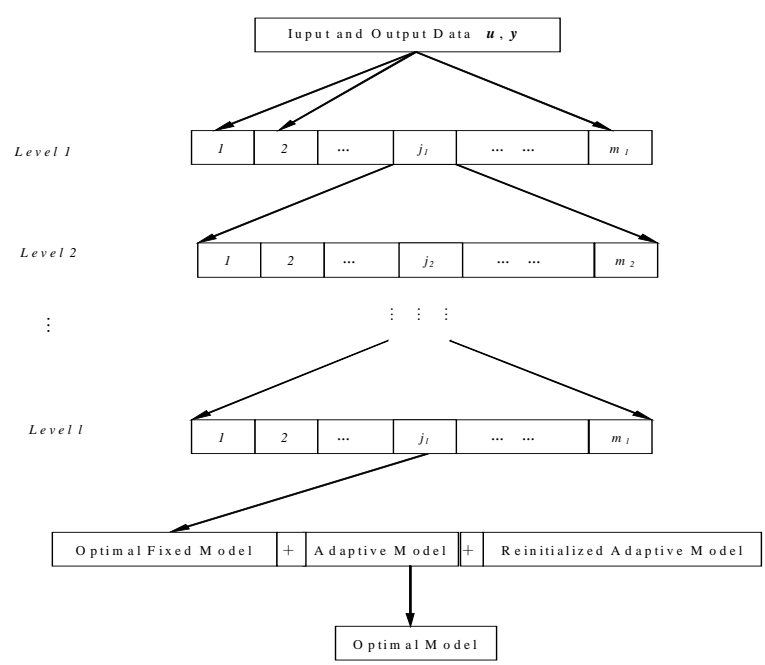

Fig. 2 Hierarchical principle of the HMMADC

\subsection{Fixed Controller Models Design}

In the HMMADC, to avoid the problem of the singularity of the matrix, the direct adaptive algorithm is adopted. Like the conventional optimal controller design, the fixed controller models are derived from the fixed system models directly. The optimal controller is chosen among these multiple controller models based on the switching index.

For the system (4), the cost function to be considered is of the form

$$
\boldsymbol{J}_{c}=\left\|\boldsymbol{P}\left(z^{-1}\right) \boldsymbol{y}(t+k)-\boldsymbol{R}\left(z^{-1}\right) \boldsymbol{w}(t)+\boldsymbol{Q}\left(z^{-1}\right) \boldsymbol{u}(t)+\boldsymbol{r}\right\|^{2},
$$

where $\boldsymbol{w}(t)$ is the known reference signal, and $\boldsymbol{P}, \boldsymbol{Q}, \boldsymbol{R}, \boldsymbol{r}$ are the weighting polynomial matrixes 
respectively. Introduce the identity

$$
\boldsymbol{P}\left(z^{-1}\right)=\boldsymbol{F}\left(z^{-1}\right) \boldsymbol{A}\left(z^{-1}\right)+z^{-k} \boldsymbol{G}\left(z^{-1}\right) .
$$

In order to get unique polynomial matrixes $\boldsymbol{F}\left(z^{-1}\right)$, $\boldsymbol{G}\left(z^{-1}\right)$, the orders of $\boldsymbol{F}\left(z^{-1}\right), \boldsymbol{G}\left(z^{-1}\right)$ are chosen as

$$
n_{f}=k-1, n_{g}=n_{a}-1 \text {. }
$$

Multiplying (4) by $\boldsymbol{F}\left(z^{-1}\right)$ from the left and using (6), to minimize the cost function (5), the optimal control law and the closed loop system can be derived as follows

$$
\begin{aligned}
& \qquad \begin{array}{l}
\boldsymbol{G}\left(z^{-1}\right) \boldsymbol{y}(t)+\boldsymbol{H}\left(z^{-1}\right) \boldsymbol{u}(t)+\overline{\boldsymbol{r}}=\boldsymbol{R}\left(z^{-1}\right) \boldsymbol{w}(t), \\
{\left[\boldsymbol{P}+\boldsymbol{Q} \boldsymbol{B}^{-1} \boldsymbol{A}\right] \boldsymbol{y}(t+k)=\boldsymbol{R} \boldsymbol{w}(t)+\boldsymbol{Q} \boldsymbol{B}^{-1}(1) \boldsymbol{d}-\boldsymbol{r},} \\
\text { where } \quad \boldsymbol{H}\left(z^{-1}\right)=\boldsymbol{F}\left(z^{-1}\right) \boldsymbol{B}\left(z^{-1}\right)+\boldsymbol{Q}\left(z^{-1}\right) \\
\overline{\boldsymbol{r}}=\boldsymbol{F d}+\boldsymbol{r} .
\end{array}
\end{aligned}
$$$$
\text { where }
$$

To place poles arbitrarily, eliminate the steady state error and the effect of $\boldsymbol{d}$ exactly, let

$$
\begin{gathered}
\boldsymbol{Q}\left(z^{-1}\right)=\boldsymbol{R}_{1} \boldsymbol{B}\left(z^{-1}\right), \\
\boldsymbol{P}\left(z^{-1}\right)+\boldsymbol{R}_{1} \boldsymbol{A}\left(z^{-1}\right)=\boldsymbol{T}\left(z^{-1}\right), \\
\boldsymbol{r}=\boldsymbol{R}_{1} \boldsymbol{d}, \\
\boldsymbol{R}\left(z^{-1}\right)=\boldsymbol{T}\left(z^{-1}\right),
\end{gathered}
$$

where $\boldsymbol{R}_{1}$ is a constant matrix. The polynomial matrix $\boldsymbol{T}\left(z^{-1}\right)$ is assumed to be stable and have the form

$$
\boldsymbol{T}\left(z^{-1}\right)=\boldsymbol{I}+\boldsymbol{T}_{1} z^{-1}+\cdots+\boldsymbol{T}_{n_{t}} z^{-n_{t}},
$$

where $\boldsymbol{T}_{\boldsymbol{i}}$ is a diagonal matrix which is decided by the designer. By the choice of weighting polynomial matrixes (10)-(13), it not only decouples the system dynamically but also places the poles of the system arbitrarily.

\subsection{Hierarchical Multiple Models Adaptive Decoupling Controller Design}

In the level $l+1$, the HMMADC is composed of three models. One is the fixed controller model $\underset{l+1,1}{\Theta}$, i.e. the best model $j_{l}$ in level $l$, the others are a free-running adaptive controller model $\underset{l+1,2}{\Theta}$ and a re-initialized adaptive controller model $\underset{l+1,3}{\Theta}$.

To the adaptive controller models $\underset{l+1,2}{\Theta}, \underset{l+1,3}{\Theta}$, Multiplying (4) by $\boldsymbol{F}\left(z^{-1}\right)$ from the left and using (6), it follows that

$$
\begin{aligned}
\boldsymbol{P}\left(z^{-1}\right) \boldsymbol{y}(t+k)= & \boldsymbol{G}\left(z^{-1}\right) \boldsymbol{y}(t) \\
& +\boldsymbol{F}\left(z^{-1}\right) \boldsymbol{B}\left(z^{-1}\right) \boldsymbol{u}(t)+\boldsymbol{F}(1) \boldsymbol{d} .
\end{aligned}
$$

Multiplying (4) by $\boldsymbol{R}_{1}$ from the left and using (11), (15), it follows that

$$
\begin{aligned}
\boldsymbol{T}\left(z^{-1}\right) \boldsymbol{y}(t+k)= & \boldsymbol{P}\left(z^{-1}\right) \boldsymbol{y}(t+k)+\boldsymbol{R}_{1} \boldsymbol{A}\left(z^{-1}\right) \boldsymbol{y}(t+k) \\
= & \boldsymbol{G}\left(z^{-1}\right) \boldsymbol{y}(t)+\boldsymbol{F}\left(z^{-1}\right) \boldsymbol{B}\left(z^{-1}\right) \boldsymbol{u}(t) \\
& +\boldsymbol{R}_{1} \boldsymbol{B}\left(z^{-1}\right) \boldsymbol{u}(t)+\boldsymbol{F}(1) \boldsymbol{d}+\boldsymbol{R}_{\mathbf{1}} \boldsymbol{d} .
\end{aligned}
$$

Using (10), (12) and the definitions of $\boldsymbol{H}\left(z^{-1}\right), \overline{\boldsymbol{r}}$, the recursive estimation algorithm of $\underset{m+1}{\Theta}$ and $\underset{m+2}{\Theta}$ is described as follows

$$
\begin{gathered}
\boldsymbol{T y}(t+k)=\boldsymbol{G} \boldsymbol{y}(t)+\boldsymbol{H u}(t)+\overline{\boldsymbol{r}}, \\
\hat{\theta}_{i}(t)=\hat{\theta}_{i}(t-1)+a(t) \frac{\boldsymbol{X}(t-k)}{1+\boldsymbol{X}(t-k)^{T} \boldsymbol{X}(t-k)} \\
\cdot\left[y_{f_{i}}(t)^{T}-\boldsymbol{X}(t-k)^{T} \hat{\theta}_{i}(t-1)\right],
\end{gathered}
$$

where $y_{f i}=T_{i i}\left(z^{-1}\right) y_{i}(t)$ is the auxiliary system output, $\quad \boldsymbol{X}(t)=\left[\boldsymbol{y}(t)^{\mathrm{T}}, \cdots ; \boldsymbol{u}(t)^{\mathrm{T}}, \cdots, 1\right]^{\mathrm{T}}$ is the data vector, $\Theta=\left[\theta_{1}, \cdots, \theta_{n}\right]$ is the controller parameter matrix

and $\theta_{i}=\left[g_{i 1}^{0}, \cdots, g_{i n}^{0} ; g_{i 1}^{1}, \cdots, g_{i n}^{1}, \cdots ; h_{i 1}^{0}, \cdots, h_{i n}^{0} ; \cdots\right]^{\mathrm{T}}$, $i=1,2, \cdots, n$. The scalar $a(t)$ is set to avoid the singularity problem of the estimation $\hat{\boldsymbol{H}}(0)$, i.e. $\hat{\boldsymbol{B}}(0)$. If $\hat{\boldsymbol{H}}(0)$ is singular and $\boldsymbol{u}(t)$ cannot be achieved, let $a(t)$ equal to another constant value in the interval $\sigma<a(t)<2-\sigma, \quad 0<\sigma<1 \quad$ to estimate $\hat{\boldsymbol{H}}(0)$ again (Goodwin et al., 1980).

To a HMMADC, the switching index is as follows

$$
J=\frac{\left\|\boldsymbol{e}_{i, s}(t)\right\|^{2}}{1+\boldsymbol{X}(t-k)^{\mathrm{T}} \boldsymbol{X}(t-k)}=\frac{\left\|\boldsymbol{y}_{f}(t)-\boldsymbol{y}_{f}(t)\right\|_{i, s}^{2}}{1+\boldsymbol{X}(t-k)^{\mathrm{T}} \boldsymbol{X}(t-k)},
$$

where $\boldsymbol{y}_{f}(t)=\boldsymbol{T}\left(z^{-1}\right) \boldsymbol{y}(t)$ is the auxiliary output of system, $\boldsymbol{e}_{f}(t)$ is the auxiliary output error between the real system and the model $s$ in level $i$. For level $1 \quad$ to $l$ let $j_{i}=\arg \min (\underset{i, s}{J}) \quad s=1, \cdots, m_{i}, \quad i=1,2, \cdots, l$

correspond to the model whose auxiliary output error is minimum , then $\underset{j}{\Theta}$ is chosen to be the best controller in level $i$. But for the level $l+1$, there are only three models left. So let $j_{l+1}=\arg \min (\underset{l+1, s}{J}) \quad s=1,2,3$, then $\underset{j+1}{\Theta}$ is chosen to be the HMMADC and used to control the system.

(1) If $j_{l+1} \neq 3$, which means $\underset{l+1,3}{\hat{\Theta}}(t)$ is not the minimum output error controller, then re-initialize $\underset{l+1,3}{\hat{\Theta}}(t)$ as the optimal controller parameter to improve the transient response, i.e. $\underset{l+1,3}{\hat{\Theta}}(t)=\underset{l+1, j_{l+1}}{\Theta}$. $\hat{\Theta}(t+1,2), \underset{l+1,3}{\hat{\Theta}}(t)$ are estimated using (18) respectively and the controller is set as $\Theta(t)=\underset{l+1, j_{l+1}}{\Theta}$.

(2) If $j_{l+1}=3, \underset{l+1,2}{\hat{\Theta}}(t), \underset{l+1,3}{\hat{\Theta}}(t)$ are estimated using (18) respectively and the controller is set as $\hat{\boldsymbol{\Theta}}(t)=\hat{\Theta}(t)$.

The optimal control law can be obtained from

$$
\hat{\boldsymbol{G}}\left(z^{-1}\right) \boldsymbol{y}(t)+\hat{\boldsymbol{H}}\left(z^{-1}\right) \boldsymbol{u}(t)+\hat{\overline{\boldsymbol{r}}}=\boldsymbol{R}\left(z^{-1}\right) \boldsymbol{w}(t) .
$$

\section{GLOBAL CONVERGENCE ANALYSIS}

Theorem 1: Subject to the assumptions 1)-4), if the algorithm (20) is applied to the system (4), 
$\{\boldsymbol{y}(\boldsymbol{t})\},\{\boldsymbol{u}(\boldsymbol{t})\}$ are bounded and $\lim _{t \rightarrow \infty}\|\boldsymbol{e}(t)\|=0$.

\section{APPLICATION TO THE WIND TUNNEL SYSTEM}

The wind tunnel system (2) is of second order and the time delay equals to 4 . Every 60 steps, the Mach number in the test section varies from 0.3 to 1.2 with $\Delta M=0.1$, which causes the parameters of the system jump simultaneously. Because the sampling period is selected as 0.1 second, 1 second in experiment means 10 steps in the simulation. The stagnation total pressure is required to be 1.5 all the time.

Case 1: A conventional adaptive decoupling controller is designed to control the wind tunnel. Its initial value is chosen close to the real controller parameter model. The responses of the system are shown in Fig. 3 and 4. In the initial stage, after 7 seconds' operation, the overshoots of the system are all less than $0.2 \%$, which satisfies the requirement. But in the experiment stage, after 0.8 second's operation, the overshoots of the system are much larger than $0.2 \%$. The largest overshoot is $68.74 \%$, which 340 times the requirement. In fact, during all experiment period, i.e. after the initial stage, the overshoots of the system are all much larger than $0.2 \%$. So the adaptive controller cannot satisfy the requirement and be used to control the wind tunnel.

Case 2: A multiple models adaptive decoupling controller is designed to control the wind tunnel. In this case, 30 fixed models are used to cover the region where jumping parameters vary. Note that the real system model is not among these fixed system models. Then 30 corresponding fixed controller models are set up using the transformation proposed above and two adaptive controller models are added to compose the multiple controller models. These two adaptive controller models' initial values are same as those of the adaptive model in case 1 . The responses of the system are shown in Fig. 5 and 6. Compared with that in case 1 , the transient response of the wind tunnel is improved greatly when only 30 fixed models are added. In the initial stage, the overshoots of the system are all less than $0.2 \%$, which satisfies the requirement. However, in the experiment stage, the overshoots of the system are all larger than $0.2 \%$, especially the stagnation total pressure (see Fig.6).

Case 3: A multiple models adaptive decoupling controller with 1000 fixed models is designed to control the wind tunnel. It is designed using the same algorithm as in case 2 but the number of the fixed models. As the number of the fixed models increases, the transient response becomes better. Both in the initial stage and in the experiment stage, the overshoots of the system are all less than $0.2 \%$, which satisfies the requirement (see Fig.7 and 8).

Case 4: A HMMADC is designed to control the wind tunnel. In this case, the same algorithm is used as in

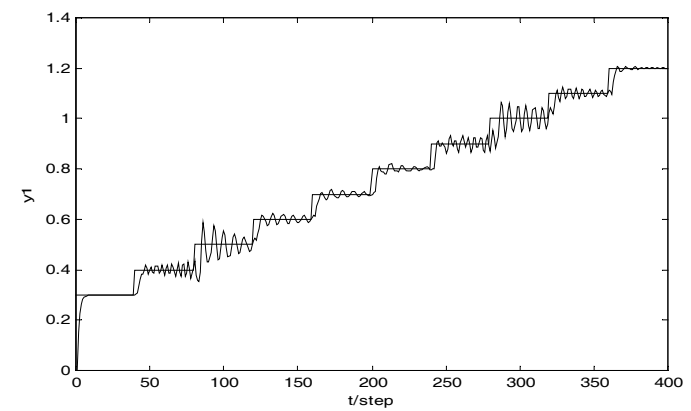

Fig.3 The Test-section-Mach-number using ADC

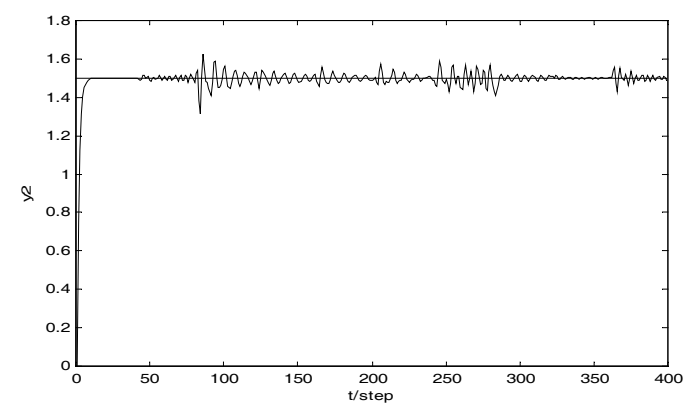

Fig.4 The Stagnation-total-pressure using ADC

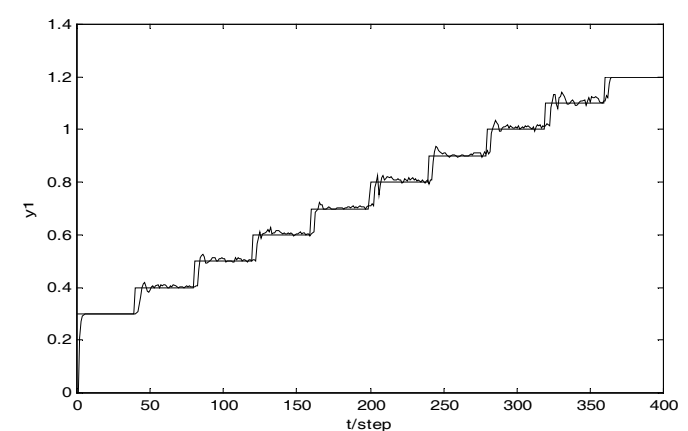

Fig.5. The Test-section-Mach-number of MMADC using 30 models

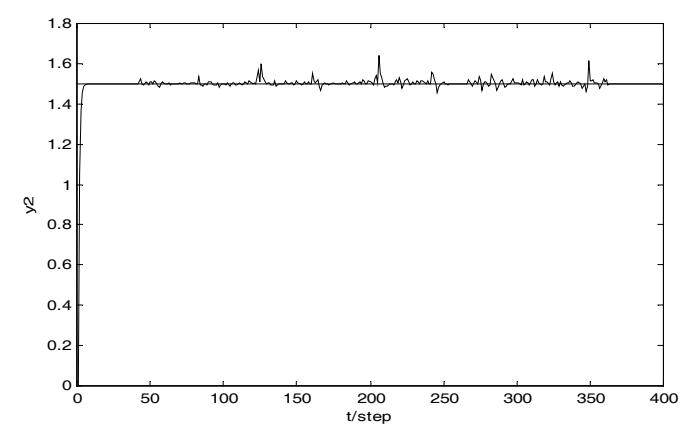

Fig.6 The Stagnation-total-pressure of MMADC using 30 models

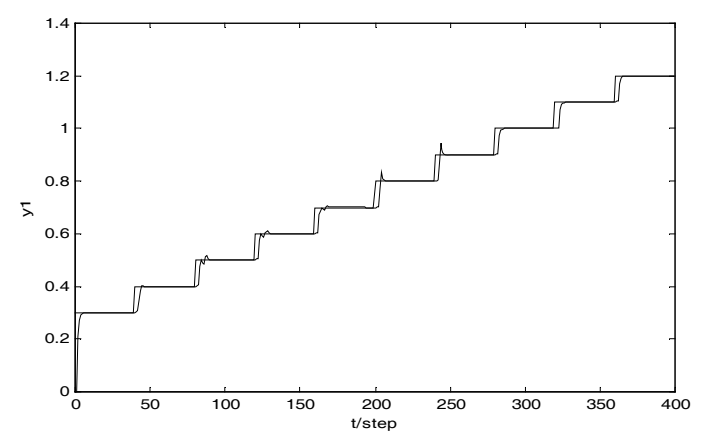

Fig.7. The Test-section-Mach-number of MMADC with 1000 models 


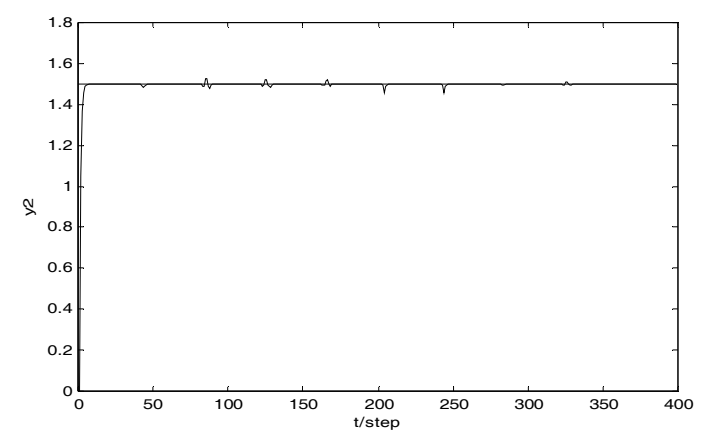

Fig.8 The Stagnation-total-pressure of MMADC using 1000 models

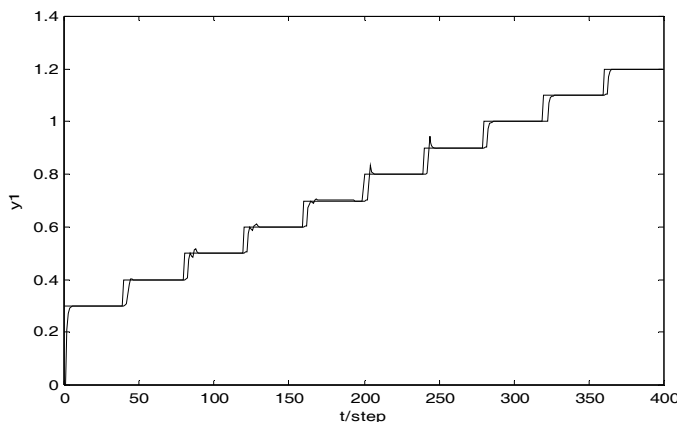

Fig.9. The Test-section-Mach-number of HMMADC using 10,10,10 models

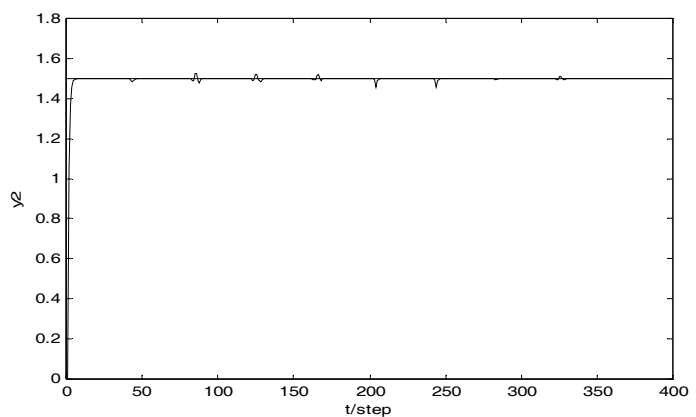

Fig.10. The Stagnation-total-pressure of HMMADC using 10,10,10 models

case 2 and 3 except a hierarchical structure with 3 levels and 10 models at each level adopted. Totally there are 30 fixed models added, the same number as in case 2, but the overshoots of the system are much better than those in case 2 . They are similar to those in case 3 , all less than $0.2 \%$, which satisfies the requirement both in the initial stage and in the experiment stage. But the number is 33 times less than that in case 3 (see Fig.9 and 10).

\section{CONCLUSION}

This paper presents a Hierarchical multiple models adaptive decoupling controller. Compared with the MMADC, the better transient response can be got with much fewer models, which reduce the number of the fixed models greatly.

\section{ACKNOWLEDGEMENT}

This work was supported by the National Natural Science Foundation of China (60474051, 70201004), National 863 High Technology Project (2003AA412310), the China Postdoctoral Science Foundation (20040350130), the Specialized Research Fund for the Doctoral Program of Higher
Education of China (20020248028), the Key Technology and Development Program of Shanghai Science and Technology Department (04DZ11008) and the Municipal Technological Super-Star Project of Shanghai (04QMX1429).

\section{REFERENCES}

CARDC (2002), Measurement and Control System Design in High and Low Speed Wind Tunnel, National Defence Industry Press, Beijing (in Chinese).

Goodwin G.C, P.J. Ramadge and P.E. Caines (1980), Discrete time multivariable adaptive control. IEEE Trans. on AC, 25, 449-456.

Landau I D, Lozano R (1981), Unification of discrete time explicit model reference adaptive control designs. Automatica, 17, 593-611

Maybeck P.S. and K.P. Hentz (1987), Inverstigation of Moving Bank Multiple Model Adaptive Algorithms, Journal of Guidance Control Dynamics. 10, 90-96.

Motter M.A and J.C. Principe (1997), Neural Control Of The NASA Langley 16-Foot Transonic Tunnel, Proceedings of the American Control Conference, $662-663$

Narendra K.S., J. Balakrishnan and M.K. Ciliz (1995), Adaptation And Learning Using Multiple Models, Switching, And Tuning. IEEE Control Systems Magazine, 15, 37-51.

Narendra K.S. and C. Xiang (2000), Adaptive Control Of Discrete-Time Systems Using Multiple Models. IEEE Trans. on AC, 45, 1669-1686.

Nelson D.M. (1989). Wind Tunnel Computer Control System and Instrumentation, Instrument Society of America, 28, 87-101.

Pels A.F.(1989). Closed-Loop Mach Number Control In A Transonic Wind Tunnel, Journal A, 30, 25-32.

Soeterboek R.A.M., A.F. Pels, etc.(1991). A Predictive Controller For The Mach Number In A Transonic Wind Tunnel. IEEE Control Systems Magazine, 11, $63-72$.

Wang X., H. Yue, et al. (2002). Multivariable Direct Adaptive Decoupling Controller Using Multiple Models, the 4th Asian Control Conference, Singapore

Wang X. S.Y. Li et al. (2005), Multiple Models Direct Adaptive Controller Applied to the Wind Tunnel System, ISA Transactions, to be published in 2005.

Yu W. and G.J. Zhang (1997). Modelling And Controller Design For 2.4 M Injector Powered Transonic Wind Tunnel, Proceedings of the American Control Conference, 1544 -1545.

Zhang G.J., T.Y. Chai and C. Shao (1997). A Synthetic Approach For Control Of Intermittent Wind Tunnel, Proceedings of the American Control Conference, 203 -207.

Zhivoglyadov P.V., R.H. Middleton and M.Y. Fu (2000), Localization Based Switching Adaptive Control For Time-Varying Discrete-Time Systems, IEEE Trans. on AC. 45, 752-755. 